\title{
PROGRESS ON MUON IONIZATION COOLING DEMONSTRATION WITH MICE
}

\author{
Paul Bogdan Jurj* \\ Imperial College London \\ E-mail: paul.jurj13@imperial.ac.uk \\ Chris Hunt \\ CERN \\ E-mail: christopher.hunt@cern.ch \\ for the MICE collaboration
}

The Muon Ionization Cooling Experiment (MICE) at the Rutherford Appleton Laboratory has collected extensive data to study the ionization cooling of muons. Several million individual particle tracks have been recorded passing through a series of focusing magnets in a number of different configurations and a liquid hydrogen or lithium hydride absorber. Measurement of the tracks upstream and downstream of the absorber has shown the expected effects of $4 \mathrm{D}$ emittance reduction. Further studies are providing more and deeper insight.

European Physical Society Conference on High Energy Physics - EPS-HEP2019 -

10-17 July, 2019

Ghent, Belgium

${ }^{*}$ Speaker. 


\section{Introduction}

Stored muon beams have been proposed as the source of neutrinos at the Neutrino Factory and as the means to deliver multi-TeV lepton-antilepton collisions at the Muon Collider [1]. In such facilities the tertiary muon beam occupies a large volume in phase space. To optimise the muon intensity, while maintaining a suitably small aperture in the muon-acceleration systems, requires that the muon-beam phase space is reduced (cooled) prior to acceleration. The short muon lifetime makes traditional cooling techniques unacceptably inefficient when applied to muon beams. Ionization cooling, in which the muon beam is passed through material (an absorber) and subsequently accelerated, is the technique by which it is proposed to cool the beam [2,3]. The MICE experiment has presented results demonstrating that ionization cooling works and is in agreement with current Monte Carlo Simulations [4].

The MICE experiment consists of an upstream beamline [5] to capture pions emitted from the titanium target [6], and focus the produced muons into a cooling channel. The cooling channel (Fig. 1) consists of 12 individually powered solenoid magnets, symmetrically placed up- and down-stream of an absorber chamber which could be configured depending on the beam momentum and required beta-function. Upstream and downstream Particle ID (PID) detectors [7] are used to improve the reconstruction algorithms and reject pion and electron contamination within the beam. A range of absorbers were using during data taking including an empty drift space (No absorber), a $65 \mathrm{~mm}$ lithium hydride disk ( $\mathrm{LiH})$ and a 221 liquid hydrogen vessel (LH2).

\section{Emittance and Amplitude}

The experiment's timing, particle identification and tracking detectors allowed individual muon tracks to be identified and reconstructed to a precision better than $1 \mathrm{~mm}$, both upstream and downstream of the absorber module. Each muon candidate was selected based on time of flight and momentum. The offline combination of the individual muon tracks permitted analysis of the collective beam behaviour. A variable thickness diffuser was included to incrementally increase the initial beam emittance between approximately $3 \mathrm{~mm}$ and $10 \mathrm{~mm}$.

The muon beam emittance was calculated by constructing the covariance matrix, $\Sigma$, using the covariances, $\sigma_{a b}$, of the position and momentum components of the individual muon tracks,

$$
\Sigma=\left(\begin{array}{cccc}
\sigma_{x x} & \sigma_{x p_{x}} & \sigma_{x y} & \sigma_{x p_{y}} \\
\sigma_{p_{x} x} & \sigma_{p_{x} p_{x}} & \sigma_{p_{x} y} & \sigma_{p_{x} p_{y}} \\
\sigma_{y x} & \sigma_{y p_{x}} & \sigma_{y y} & \sigma_{y p_{y}} \\
\sigma_{p_{y} x} & \sigma_{p_{y} p_{x}} & \sigma_{p_{y} y} & \sigma_{p_{y} p_{y}}
\end{array}\right) .
$$

The 4-dimensional normalized transverse emittance, $\epsilon_{4 D}$, of the beam can then be calculated using the determinant of the covariance matrix and the muon mass, $m_{\mu}$ :

$$
\epsilon_{4 D}=\frac{1}{m_{\mu}} \sqrt[4]{|\Sigma|}
$$

The single-particle amplitude, $A_{\perp}$, at a point $v=\left(x, p_{x}, y, p_{y}\right)$ in phase space can be defined as the square of the distance between $v$ and the centre of the distribution, normalised to the covariance 

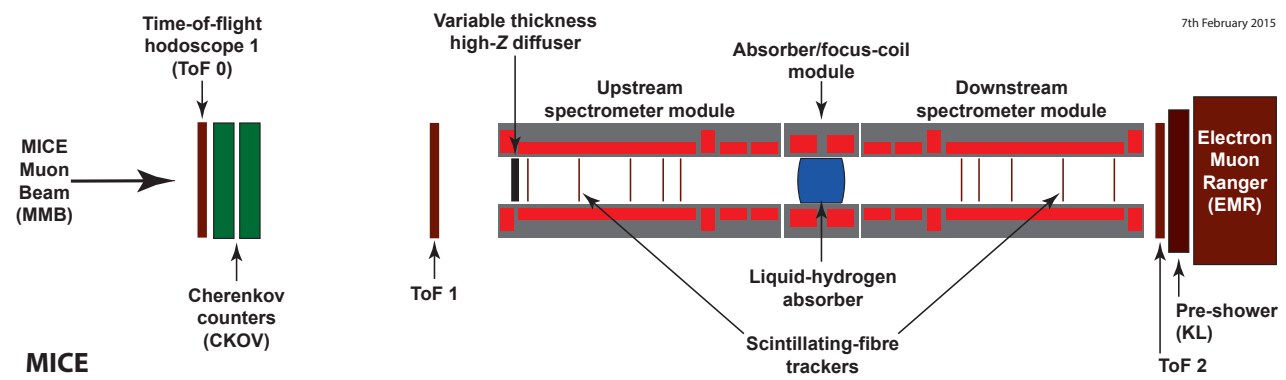

Figure 1: Schematic layout of the MICE cooling channel. Magnet coils are shown in red, the absorber in blue, and the various detectors are individually marked.

matrix and weighted by the distribution's emittance. It estimates the emittance of a beam which is characterized by an ellipse that passes through that point. It is calculated as

$$
A_{\perp}=\epsilon_{4 D}(v-\bar{v})^{T} \Sigma^{-1}(v-\bar{v})
$$

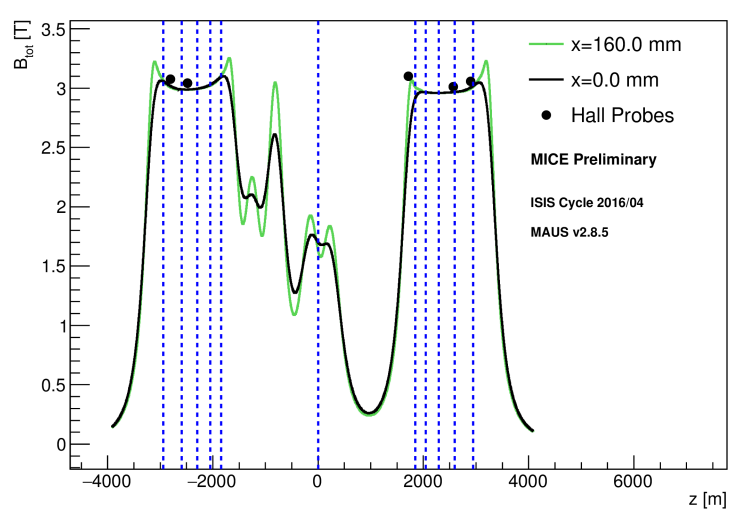

Figure 2: Magnitude of the of the magnetic field along the length of the cooling channel. Hall probe measurements are included as verification, and the field strength at the position of the Hall probes is shown in green (160 mm off axis). The central cusp is due to the magnetic field flip.

\section{Method}

Data were taken with a specific configuration of solenoidal fields, i.e., $\beta \sim 660 \mathrm{~mm}$ at the absorber, with the three different absorber configurations (No absorber, LiH and LH2). The magnitude of the solenoidal field strength along the length of the cooling channel is shown in Fig. 2. Scintillating fiber detectors up- and downstream of the absorber were used to reconstruct individual muon tracks [8][9]. The data collected using an empty channel enabled the observation of optical aberrations and will be used to cross-check for other systematic effects.

Cuts were applied to the raw data to remove electron and pion impurities in the reconstructed beam. The recorded time of flight for each track was required to be consistent with the expected value given the selected muon momentum $(140 \pm 7 \mathrm{MeV})$. A well-reconstructed track was required in the upstream and, if present, the downstream trackers $\left(\chi^{2} / \mathrm{NDF}<10\right)$. Each track was also 
required to be well contained within the tracking volume and not be incident on any hard apertures that could cause scraping.

The full ensemble of tracks that passed all the cuts and were successfully reconstructed in the upstream tracker were combined to produce the upstream sample. Those tracks that were transmitted to the downstream tracker and were well-reconstructed were combined to form the downstream sample. Each sample was analyzed independently to calculate the covariance matrix and the amplitude distributions.

a)
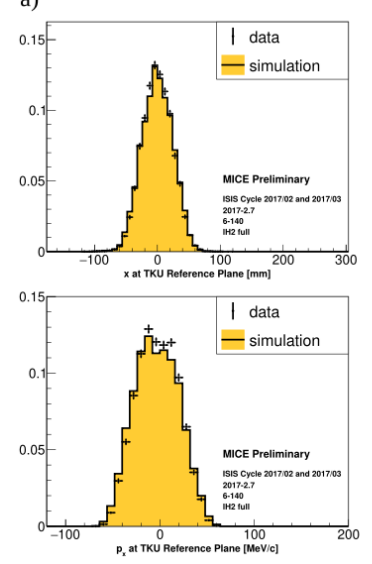
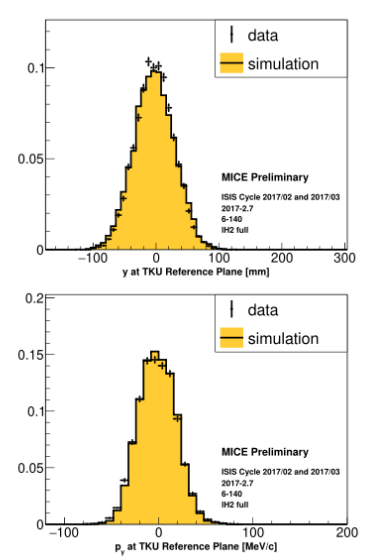

b)
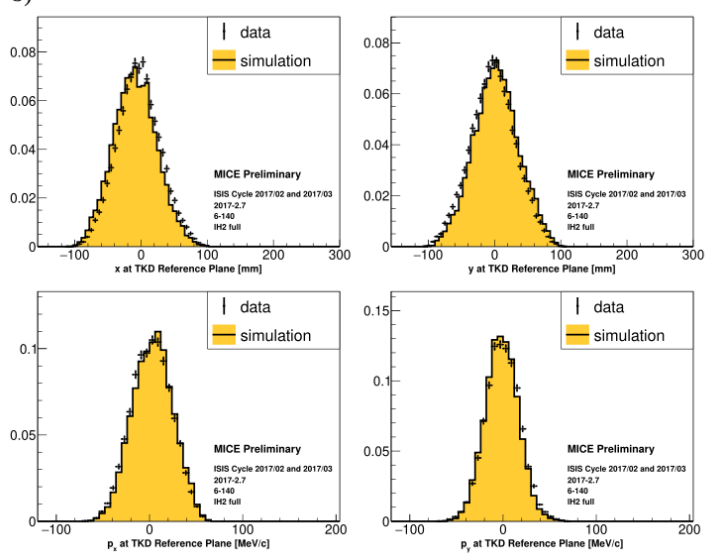

Figure 3: Distributions of the upstream (a) and downstream (b), position (top) and momentum (bottom) parameters in $x$ (left) and $y$ (right). All show good agreement between data and simulation.

\section{Analysis}

The upstream and downstream samples were evaluated following the selection and reconstruction procedures and are shown in Fig. 3. There is close agreement between simulation and data, showing that the experiment is well understood and in agreement with the current Monte Carlo models.

The amplitude of each muon in the up- and downstream samples was calculated using Eq. (2.3). The distribution of amplitudes for the up- and downstream samples was integrated from zero, bin-by-bin, to produce cumulative distributions. The ratio of the cumulative distributions for two initial emittances (6 and $10 \mathrm{~mm}$ ) and three absorber configurations is shown in Fig. 4.

Bins above (below) 1 indicate particle migration into (out of) that area of phase-space. The No absorber case demonstrates the effects of scraping - medium/high amplitude particles migrating outwards and being lost - and the final transmission is less than 1 , as seen by the final bin. The core of the beam undergoes no significant change in this case.

In the $\mathrm{LH} 2$ and $\mathrm{LiH}$ cases, a clear increase in particle density is seen in the core of the beam, a signal which is a direct consequence of the absorber being present within the cooling channel. As expected, the $10 \mathrm{~mm}$ beam sees a larger effect due to cooling than the $6 \mathrm{~mm}$ beam, while the transmission is lower ( $\sim 94 \%$ versus $\sim 98 \%$ ), due to increased scraping. These results demonstrate the migration of high amplitude particles to lower amplitudes, significantly above any statistical fluctuations within the distributions. 


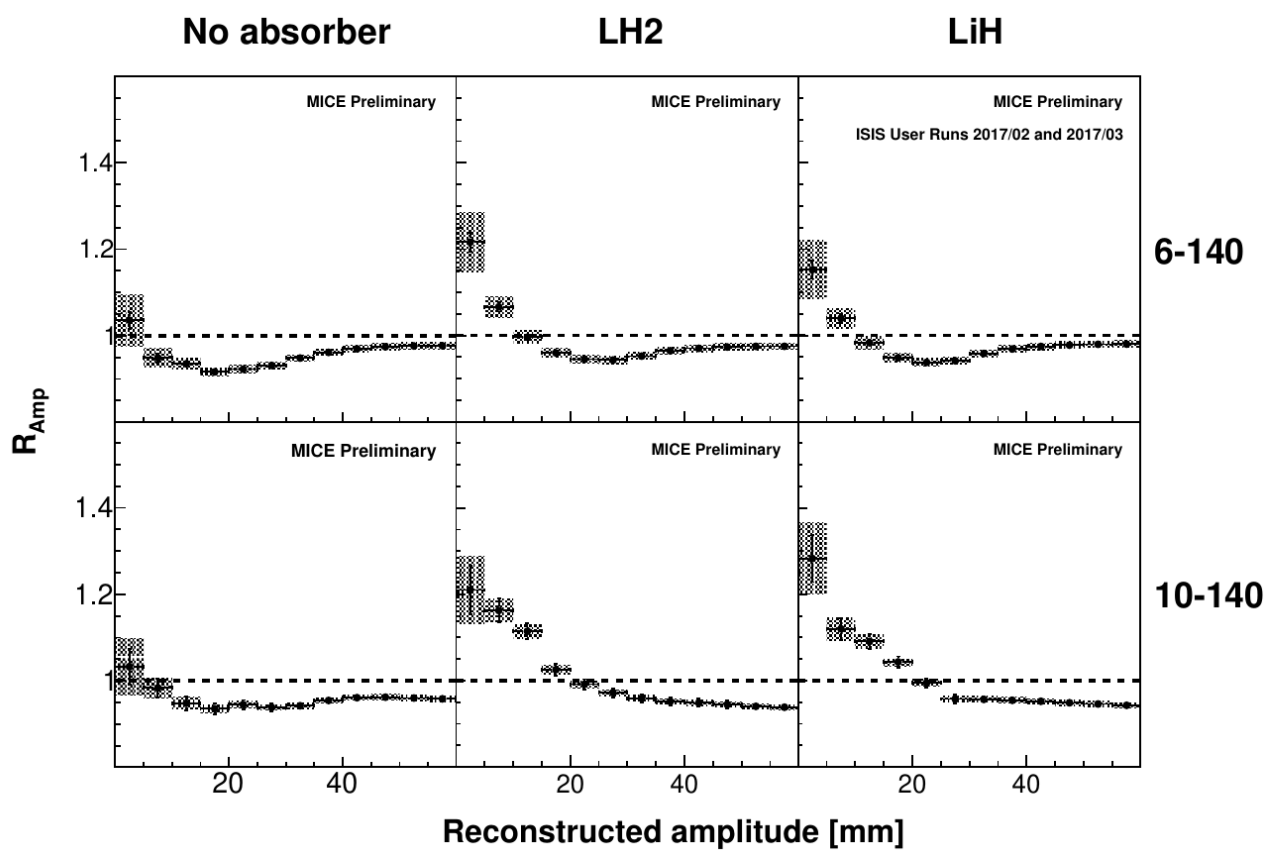

Figure 4: Comparison of the ratio of cumulative amplitude distributions, $R_{A m p}$, for the two initial emittances and three absorber configurations. Bins that deviate from a flat distribution demonstrate areas of migration. Migration towards the centre of the beam can only be produced through ionization cooling. The value in the final bin indicates the transmission of the particular configuration.

\section{Conclusion}

The MICE experiment has successfully demonstrated that the interaction between a muon beam and a low- $Z$ absorber can be used to stimulate a migration in particle amplitudes towards the core of the beam. This is a signal uniquely indicative of ionization cooling. The Monte Carlo simulations have been shown to be in good agreement with the data, hence the observed results are in agreement with existing material models used in the Geant 4 simulation framework. It is anticipated that the refinement of our beam selection routines will further reduce the effects of optical aberrations and permit the measurement of an enhanced cooling signal.

\section{Acknowledgements}

The work described here was made possible by grants from Department of Energy and National Science Foundation (USA), the Istituto Nazionale di Fisica Nucleare (Italy), the Science and Technology Facilities Council (UK), the European Community under the European Commission Framework Programme 7 (AIDA project, grant agreement no. 262025, TIARA project, grant agreement no. 261905, and EuCARD), the Japan Society for the Promotion of Science and the Swiss National Science Foundation, in the framework of the SCOPES programme. We gratefully acknowledge all sources of support. We are grateful to the support given to us by the staff of the STFC Rutherford Appleton and Daresbury Laboratories and the Cockroft Institute. We 
acknowledge the use of Grid computing resources deployed and operated by GridPP in the UK, http://www.gridpp.ac.uk/.

\section{References}

[1] S. Geer, "Neutrino beams from muon storage rings: Characteristics and physics potential", Phys. Rev. $D$, vol. 57 , no. 11 , p. 6989, 1998.

[2] A. N. Skrinsky et al., "Cooling methods for beams of charged particles", Sov. J. Part. Nucl., vol. 12, no. 223, 1981.

[3] D. Neuffer, "Principles and Applications of Muon Cooling", Part. Accel., vol. 14, pp. 75-90, 1983.

[4] T. Mohayai, "First demonstration of ionization cooling in MICE," in Proc. 9th Int. Particle Accelerator Conf. (IPAC'18), 2018. doi : 10.18429/JACoW-IPAC2018-FRXGBE3.

[5] M. Bogomilov et al., "The MICE Muon Beam on ISIS and the beam-line instrumentation of the Muon Ionization Cooling Experiment”, JINST, vol. 7, p. 5009, 2012.

[6] C. N. Booth et al., "The design, construction and performance of the MICE target", JINST, vol. 8. 2013.

[7] D. Orestano, “The detector system of the MICE experiment”, Nucl. Inst. Phys. A, vol. 617, pp. 45 47, 2010.

[8] M. Ellis et al., "The design, construction and performance of the MICE scintillating fibre trackers", NIM, A 659, 136, 2011.

[9] A. Dobbs et al., "The reconstruction software for the mice scintillating fibre trackers", Journal of Instrumentation, vol. 11, no. 12, p. T12001, 2016. 